\title{
The Sensitivity and Specificity of Fine- Needle Aspiration in Thyroid Neoplasia
}

\author{
Rareș Georgescuํ, Adela Luciana Oprea², Alexandra Contra1, Orsolya Bauer Hanko', Ioana \\ Colcer ${ }^{1}$, Marius Florin Coroș ${ }^{1}$ \\ ${ }^{1}$ Department of Surgery, University of Medicine and Pharmacy, Tîrgu Mureș, Romania \\ ${ }^{2}$ Department of Obstetrics and Gynecology, University of Medicine and Pharmacy, Tîrgu Mureș, Romania
}

\section{CORRESPONDENCE}

Adela Luciana Oprea

Str. Gheorghe Marinesu nr. 50

540136 Tîrgu Mureș, Romania

Tel +40 743859357

E-mail: adela_luciana_oprea@yahoo.com

\section{ARTICLE HISTORY}

Received: 29 March, 2017

Accepted: 30 May, 2017
Rareș Georgescu • Str. Gheorghe Marinesu nr. 38 540139 Tîrgu Mureș. Romania, Tel: +40 265215551. E-mail: rares1geo@yahoo.com

Alexandra Contra • Str. Gheorghe Marinesu nr. 38 540139 Tîrgu Mures. Romania, Tel: +40 265215551. E-mail:

Orsolya Bauer Hanko • Str. Gheorghe Marinesu nr. 38, 540139 Tîrgu Mureș. Romania, Tel: +40 265215 551. E-mail: orsolyabauer@gmail.com

loana Colcer • Str. Gheorghe Marinesu nr. 38, 540139 Tîrgu Mureș. Romania, Tel: +40 265215 551. E-mail: colcer_ioana@yahoo.com

Marius Florin Coroș • Str. Gheorghe Marinesu nr. 38 540139 Tîrgu Mureș. Romania, Tel: +40 265215551.

E-mail:mcoros@gmail.com

\begin{abstract}
Objective: To evaluate and demonstrate the accuracy of fine-needle aspiration (FNA) in thyroid lesions in our department and to highlight probable causes of errors leading to unsatisfactory sampling, which may depend on the characteristics of the nodule. Methods: This is a retrospective study conducted on 319 diagnosed cases of thyroid nodules referred to the Surgery Unit of Puls hospital, Tîrgu Mureș in the January 2014 - December 2015 period, who underwent fine-needle aspiration. Histological examination was considered to be the gold standard; therefore we compared the cytological diagnosis with the histological one. Results: Of the 319 cases, 289 (90.6\%) were female and 30 (9.4\%) male patients; 210 cases (69.3\%) were interpreted as benign, 46 cases (15.2\%) as follicular lesion of undetermined significance, 4 cases (1.3\%) as suspect for malignancy, 1 case (0.3\%) as malignant sampling, and 42 cases (13.9\%) as unsatisfactory. We compared the results of fine-needle aspiration cytology (FNAC) with the corresponding histopathological results (49 in total). FNAC achieved a sensitivity of $76.47 \%$, a specificity of $83.1 \%$, a positive predictive value of $35.1 \%$, a negative predictive value of $96.7 \%$, a false positive rate of $16.9 \%$, a false negative rate of $23 \%$, and an overall accuracy of $82.3 \%$. Conclusions: The results of our study demonstrate the accuracy of the FNA technique in the first-line diagnosis of thyroid nodules.
\end{abstract}

Keywords: FNA, thyroid cancer, sensitivity, specificity

\section{INTRODUCTION}

Thyroid nodules are a common clinical finding in the general population, their prevalence at palpation ranging from $3 \%$ to $7 \%$. Nowadays, ultrasound imaging is routinely used to detect thyroid or non-thyroid neck pathology, resulting in a dramatically increased number of clinically unapparent nodules, ranging from $20 \%$ to $76 \% .^{1}$ There is a large number of imaging techniques used in the diagnosis of thyroid nodules, but guidelines indicate that fine-needle aspiration cytology (FNAC) is the first-line diagnostic test, being the most accurate and costefficient procedure. These attributes are greatly enhanced when ultrasound is used as a guide for better sampling. ${ }^{2}$ Published data suggest that fine-needle aspiration (FNA) has a sensitivity ranging from $65 \%$ to $98 \%$, a specificity from 
$72 \%$ to $100 \%$, and an overall accuracy of $75 \%$ to $90 \% .^{3}$ Nevertheless, like any other test, FNAC has its limitations and diagnostic pitfalls. These limitations include false negative and false positive results, and a proportion of FNA results that are not obviously benign or malignant, fall into the indeterminate or suspicious group. ${ }^{4}$ The reported pitfalls are those related to specimen adequacy, sampling techniques, the skill of the physician performing the aspiration, the experience of the pathologist interpreting the aspirate, and the overlapping cytological features between certain benign and malignant thyroid lesions. ${ }^{5}$ As already noted, about $70 \%$ of FNA specimens are classified as benign; in addition, $5 \%$ are malignant, $10 \%$ are suspicious, and $10 \%$ to $20 \%$ are non-diagnostic or unsatisfactory. Nevertheless, in order to improve diagnostic accuracy, the unsatisfactory samples should be maintained at rates smaller than $10 \% .^{1,6}$

Our aim was to evaluate and demonstrate the accuracy of the FNAC technique in thyroid lesions at our department, and to highlight probable causes of errors leading to unsatisfactory sampling, which may depend on the characteristics of the nodule (specimen adequacy-related pitfall).

\section{MATERIALS AND METHODS}

\section{FNA technique}

First of all, FNA is explained to the patient and usually performed in local anesthesia. The patient has to lie down on her back, with her head tipped backwards, to assure that the neck is extended. A pillow can also be placed under the patient's shoulders, to get a better position. The patient is asked to stay as still as possible and to avoid speaking and swallowing during the procedure. The benign or malign nodules are identified by ultrasound. A 22 gauge needle is introduced into the thyroid nodule under ultrasound guidance. Moving the needle back and forth, the practician will take approximately 20 tissue samples. This procedure is performed twice, until enough tissue is collected. Then, two slide smears are made for each case, immediately air-dried with a warm air-drying device and sent to the laboratory. After the procedure, the nurse or the doctor should apply pressure on the neck for several minutes to avoid bleeding.

\section{Ethical considerations}

Ethical approval was obtained from the Ethics Commission of the University of Medicine and Pharmacy of Tîrgu
Mureș, and a written informed consent was waived by Puls Hospital.

\section{Patient selection and working method}

This is a retrospective study conducted on 319 diagnosed cases of thyroid nodules referred to the Surgery Unit of Puls Hospital, Tîrgu Mureș in the January 2014 - December 2015 period, in order to establish a FNAC diagnosis. The patients had ultrasound examination performed by endocrinologists in different clinics, after which, depending on the ultrasound images, they were selected and referred to our department for FNAC. All solitary nodules were selected for FNAC regardless of their sonographic and color-Doppler features. When a multinodular thyroid gland was encountered, the largest nodule was included in the study, unless an atypical nodule with ultrasound features specific for malignancy was encountered. ${ }^{7}$

Information about the patients' age, sex, ultrasound findings, and cytological diagnosis was retrieved from the case notes. Information regarding the histological diagnoses or ultrasound follow-up was retrieved by telephonic questionnaire directly from the patients.

Thyroid nodules were aspirated using disposable needles, following the standard procedures. ${ }^{8}$ Two slide smears were made for each case and immediately air-dried with a warm air-drying device. They were let unfixed and transported to the laboratory for Giemsa staining. All thyroid nodule aspirates were collected by the same surgeon, with 4 years of experience in this procedure at the time of the study. The diagnosis of cytological smears was done according to the Bethesda system for reporting thyroid cytopathology into: insufficient for diagnosis, benign, atypical follicular lesion of undetermined significance, follicular neoplasm, suspicious for malignancy, and malignant sampling. Aspirates with insufficient cellularity or poor quality smears due to delayed or inadequate fixation were considered "unsatisfactory".

Histological examination was considered to be the gold standard; therefore we compared the cytological diagnosis with the histological one. Most of the patients with benign cytological findings did not undergo surgery and they were followed-up by ultrasound imaging assessment after at least six months since the FNAC evaluation. In order to calculate the accuracy of FNAC, we did not include the unsatisfactory results in any benign or malignant categories; therefore, these cases were excluded from the study.

Cases in which we could not retrieve any further information after the FNAC procedure took place or six months have not passed since the cytological diagnosis (necessary 
to ensure the stability of the benign nodule through ultrasound), were also excluded from the calculations.

For the purpose of the study, the sonographic and color Doppler features of the nodule selected for FNAC unsatisfactory sampling analysis were categorized based on the dimensions ( $<10 \mathrm{~mm}$ or greater) and content of the nodule (cystic or not), characteristics which are regarded as problematic and may influence the quality of the sample. ${ }^{1,6} \mathrm{We}$ also considered that nodular color Doppler flow characteristics (no flow, peripheral and/or central flow) and the presence of calcification (no calcification, small $-2 \mathrm{~mm}$, or coarse $->2 \mathrm{~mm}$ calcification) may also influence the quality of the sample in different proportions (obscuring blood in case of intense vascularity, or nodule hard to aspirate in case of large calcifications). ${ }^{6}$

\section{Statistical analysis}

Receiver operating characteristic (ROC) curve analysis was used to examine the accuracy of FNAC in differentiating benign and malignant nodules and reducing unnecessary surgery. Logistic regression was used to predict the probability of a thyroid nodule sampling to turn up being inadequate due to an ultrasound characteristic of the nodule (dimension, consistency, color flow, presence of calcification). The statistical analyses were performed using MedCalc version 13.1.1 (MedCalc Software, Ostend, Belgium).

\section{RESULTS}

Out of the 319 patients, we were able to retrieve information regarding the histological diagnoses or ultrasound follow-up in 189 patients. The remaining 130 patients were excluded due to missing follow-up scans to ensure benignity or the histology findings were unavailable (surgery at another center or refused).

Of the 319 cases, 289 (90.6\%) were female and 30 (9.4\%) were male patients. The median age was 53 years (range 17-82 years).
Two-hundred ten cases (69.3\%) were interpreted as benign, 46 cases (15.2\%) as follicular lesion of undetermined significance, 4 cases (1.3\%) as suspect for malignancy, 1 case $(0.3 \%)$ as malignant sampling, and 42 cases $(13.9 \%)$ as unsatisfactory.

We compared the results of the FNAC with the corresponding histopathological ones (49 in total). Eighteen benign diagnosed cases had histopathology results (upon the endocrinologist's request in most of the cases and upon the surgeon's recommendation for symptoms relieve in others). Seventeen cases of follicular lesion of undetermined significance, 3 out of 4 cases of suspect for malignancy, the single case of malignant sampling and 10 out of 42 cases of unsatisfactory sampling also had histopathology results (Table 1).

Of the 18 benign cases who underwent surgery, 4 cases were diagnosed as carcinoma (22\%), the rest of them were nodular goiter or adenoma. Only 4 out of the 17 cases of follicular lesion of undetermined significance turned up to be malignant, the malignant sampling was confirmed as being malignant, and 1 out of the 3 suspect cases showed up to be benign. Four out of 10 unsatisfactory samplings were malignant.

All the malignant results in histopathology were papillary carcinoma, except for one case of lymphoma and one case of follicular neoplasm.

Of the 140 patients who did not undergo surgery but had ultrasonography follow-up for at least 6 months, 137 (97.9\%) showed no significant increase in size, no ultrasonographic changes, and no pressure symptoms.

Of the 122 included cases interpreted as benign in FNAC, 118 proved to be non-neoplastic (by histopathology result or ultrasound follow-up), while 3 cases were diagnosed as papillary carcinoma and 1 case as follicular neoplasm in histopathology. Out of the 37 cases interpreted as suspect for malignancy, malignant sampling, or follicular lesion of undetermined significance in FNAC, 24 cases turned up being non-neoplastic and only 13 were actually neoplastic in histopathology (Table 2).

TABLE 1. The relationship between cytological and histological findings

\begin{tabular}{|c|c|c|c|c|c|c|c|}
\hline \multirow{3}{*}{$\begin{array}{l}\text { Cytological } \\
\text { diagnosis }\end{array}$} & \multirow{3}{*}{$\begin{array}{c}\text { No. of } \\
\text { cases with } \\
\text { available } \\
\text { cytology }\end{array}$} & \multirow{3}{*}{$\begin{array}{c}\text { No. of } \\
\text { cases with } \\
\text { available } \\
\text { histology }\end{array}$} & \multicolumn{3}{|c|}{ Histological diagnosis } & \multirow[t]{3}{*}{ FP } & \multirow[t]{3}{*}{ FN } \\
\hline & & & \multirow{2}{*}{$\begin{array}{c}\text { Consistent } \\
\text { with cytology }\end{array}$} & \multicolumn{2}{|c|}{ Inconsistent with cytology } & & \\
\hline & & & & Benign & Malignant & & \\
\hline Benign & 210 & 18 & 14 & - & 4 & & 4 \\
\hline Intermediate & 46 & 17 & & & - & & \\
\hline Suspect or malign & 5 & 4 & 3 & 1 & - & 1 & \\
\hline
\end{tabular}




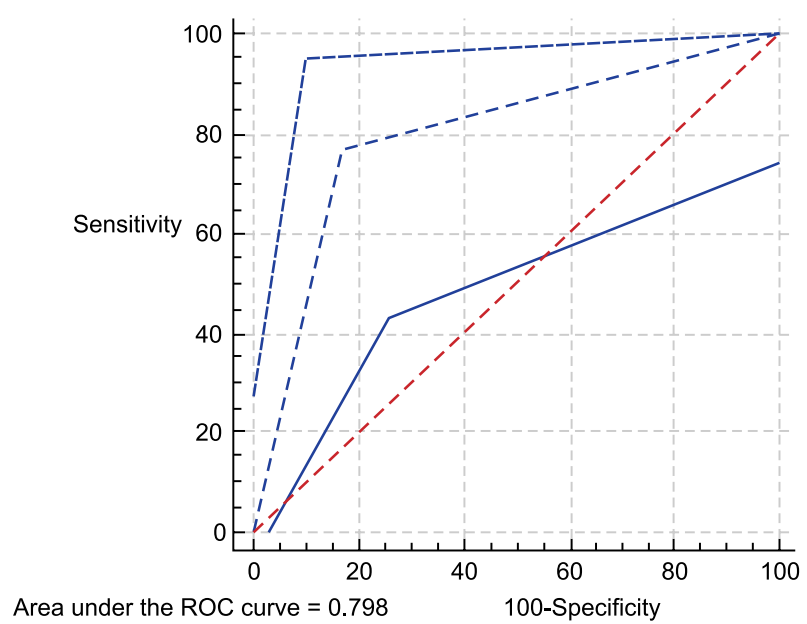

FIGURE 1. ROC curve shows high area-under-the-curve (AUC) values, indicating a good diagnostic performance in predicting the outcome

Thus, FNAC achieved a sensitivity of $76.47 \%$, a specificity of $83.1 \%$, a positive predictive value of $35.1 \%$, a negative predictive value of $96.7 \%$, a false positive rate of $16.9 \%$, a false negative rate of $23 \%$, and an overall accuracy of $82.3 \%$.

The ROC curve shows high area-under-the-curve (AUC) values (AUC $=0.798$ ), indicating a good diagnostic performance for FNAC.

The thyroid nodules varied in size, with diameters ranging from 0.3 to $5.5 \mathrm{~cm}$. There was a significant difference in size between benign nodules $(2.1 \pm 1.1 \mathrm{~cm})$ and malignant nodules $(1.6 \pm 0.8 \mathrm{~cm})(\mathrm{p}=0.001)$.

Logistic regression analysis results suggest that only the content of the nodule characteristic is significantly associated with a higher rate of sample inadequacy when aspiring thyroid nodules (cystic lesion) $(\mathrm{p}=0.04)$. The dimension of the nodule characteristic had a $\mathrm{p}$ value of 0.1 , indicating that aspiring nodules smaller than $1 \mathrm{~cm}$ did not have a statistically significant influence on the rate of inadequate sampling.

\section{DISCUSSIONS}

FNA has an essential role in the evaluation of euthyroid patients with a thyroid nodule. It reduces the rate of unnecessary thyroid surgery for patients with benign nodules and appropriately triages patients with thyroid cancer to appropriate surgery. ${ }^{6}$

Nonetheless, there are a lot of diagnostic limitations regarding FNAC, related not only to inadequate sampling, but also, most importantly, to its inability to distinguish
TABLE 2. The relationship between cytology and final diagnosis

\begin{tabular}{lccc}
\hline Cytological diagnosis & \multicolumn{2}{c}{ Final diagnosis } & \multirow{2}{*}{ Total } \\
\cline { 2 - 3 } & Benign & Malignant & \\
\hline Benign & $118(\mathrm{TN})$ & $4(\mathrm{FN})$ & 122 \\
$\begin{array}{l}\text { Suspicious, malignant, } \\
\begin{array}{l}\text { undetermined } \\
\text { Total }\end{array}\end{array}$ & $24(\mathrm{FP})$ & $13(\mathrm{TP})$ & 37 \\
\hline
\end{tabular}

between benign and malignant follicular lesions in the absence of nuclear features of papillary carcinoma. The indeterminate diagnosis of follicular neoplasm encompasses a number of heterogeneous thyroid lesions including cellular adenomatoid nodule, follicular adenoma, and follicular carcinoma. ${ }^{9}$

Regarding the inadequate sampling pitfall, we had to confront with a considerable number of unsatisfactory samplings in our study, which we were not able to report (approximately 17\%). This group also included aspirates consisting only of cyst fluid. Thus, the proportion of unsatisfactory sampling was calculated after excluding the inadequate aspirates from the cystic lesions (macrophages only), because according to the Bethesda reporting system they should be considered a clearly identified subset of ND/UNS, ${ }^{6}$ and our laboratory considered that this was not the case. The new proportion of inadequate sampling was reduced from $17 \%$ to $13.9 \%$, a percentage which is in accordance with data communicated by other studies. ${ }^{10}$

Similarly to other studies, the majority of the cases were benign. ${ }^{1}$ In contrast, the suspect for malignancy and malignant sampling cases in cytology represented a highly reduced proportion of the entire number of FNAC results, contradicting usually published data.

We are assuming that these diagnostic categories were used less than the "follicular lesion of undetermined significance" diagnosis by our cytologist. Similar situations were communicated in studies in which several cytologists described and compared the same smears. ${ }^{11}$ Thus, we are considering to have the slides reviewed by more than one cytologist.

In our study, we achieved a sensitivity of $76.47 \%$ and a specificity of $83.1 \%$, which built up into an overall accuracy of $82.3 \%$, a positive predictive value of $35.1 \%$, a negative predictive value of $96.7 \%$, a false positive rate of $16.9 \%$, and a false negative rate of $23 \%$. The results are partially comparable with other published data, which report a sensitivity ranging from $65 \%$ to $98 \%$, a specificity from $72 \%$ to $100 \%$, and an overall accuracy of $75 \%$ to $90 \% .^{3}$ 
Positive predictive values (PPV) and negative predictive values (NPV) are defined as the proportions of positive and negative results that are true positive and true negative. A low positive predictive value of $35 \%$ indicates that our procedure is lacking in truly identifying the malignant nodules. This may be due to interpreting poor specimens as follicular lesions of undetermined significance and to the reduced number of slides ( 2 colored slides per patient). Some authors suggest that at least 6 punctures should be made, and 8 to 10 slides should be colored and interpreted before deciding upon the diagnosis. ${ }^{12}$

The false positive rate (FPR) is high (16.9\%) because of the increased number of follicular lesion of undetermined cytology diagnoses, which proved to be benign at histopathology. Again, this is related to the reduced number of slides interpreted by the cytologist.

The false negative rate (FNR) is defined as the percentage of patients with benign cytology in whom malignant lesions are later confirmed on thyroidectomy. In our study we had 4 such cases, translating into a FNR of $23 \%$. Of these 4 cases, 1 was a follicular neoplasm, which is very difficult to diagnose from follicular adenomas in FNAC. ${ }^{13}$

Currently in our clinic, we offer FNAC analysis routinely in the evaluation of thyroid nodules, but recognize the need to be discerning in selecting malignant nodules for FNAC and hence reduce unnecessary FNA biopsies. ${ }^{14}$

Even if our study shows that aspiring nodules smaller than $1 \mathrm{~cm}$ did not influence in a statistically significant manner the rate of inadequate sampling, we consider it worth mentioning that out of 62 incidentalomas, 11 were not interpreted due to unsatisfactory sampling (17.7\%).

\section{CONCLUSION}

The results of our study are comparable with other data from literature, demonstrating the accuracy of the FNA technique in the first-line diagnosis of thyroid nodules.
The criteria for referring incidentalomas for FNA should be reviewed more strictly, and benign FNAC should be reviewed with caution as false negative cases, especially if there are suspicious findings in ultrasound imaging.

\section{CONFLICT OF INTEREST}

Nothing to declare.

\section{REFERENCES}

1. Gharib H, Papini E, Valcavi R, et al. American Association of Clinical Endocrinologists and Associazione Medici Endocrinologi medical guidelines for clinical practice for the diagnosis and management of thyroid nodules. Endocr Pract. 2006;12:63-102

2. Orell SR. In: Orell SR, Sterrett GF, Walters MN, Whitakar D, editors. Manual and atlas of fine needle aspiration cytology. New Delhi: ChurchillLivingstone; 2005. p. 125-164.

3. Jakowski JD. Palpation Guided Fine Needle Aspiration Analysis of FNA Procedure and Diagnosis. Available at: http://www.pathologyoutlines. com/topic/cytopathologypgfnaanalysis.html

4. Galera-Davidson H. Diagnostic problems in thyroid fine needle aspirations. Diagn Cytopathol. 1997;17:422-428.

5. Baloch ZW, Sack MJ, Yu GH, Livolsi VA, Gupta PK. Fine needle aspiration of thyroid an institutional experience. Thyroid. 1998;8:565-569.

6. Cibas ES, Ali SZ. The Bethesda System for Reporting Thyroid Cytopathology. Am J Clin Pathol. 2009;132:658-665.

7. Lingam RK, Qarib MH, Tolley NS. Evaluating thyroid nodules: predicting and selecting malignant nodules for fine-needle aspiration (FNA) cytology. Insights Imaging. 2013;4:617-624.

8. Belfiore A, La Rosa GL. Fine-needle aspiration biopsy of the thyroid. Endocrinology \& Metabolism Clinics of North America. 2001;30:361-400.

9. Saggiorato E, De PR, Volante M. Characterization of thyroid 'follicular neoplasms' in fine-needle aspiration cytological specimens using a panel of immunohistochemical markers: a proposal for clinical application. Endocr Relat Cancer. 2005;12:305-317.

10. Guhamallick M, Sengupta S, Bhattacharya NK, Chowdhury M. Cytodiagnosis of thyroid lesions-usefulness and pitfalls: A study of 288 cases. Journal of Cytology. 2008;25:6-9

11. Raab SS, Vrbin CM, Grzybicki DM, et al. Errors in Thyroid Gland FineNeedle Aspiration. Am J Clin Pathol. 2006;125:873-882.

12. Hamburger JI. Diagnosis of thyroid nodules by fine needle biopsy: use and abuse. J Clin Endocrinol Metab. 1994;79:335-339.

13. Rossi ED, Raffaelli M, Minimo C. Immunocytochemical evaluation of thyroid neoplasms on thin-layer smears from fine-needle aspiration biopsies. Cancer. 2005;105:87-95

14. Ahn SS, Kim EK, Kang DR, Lim SK, Kwak JY, Kim MJ. Biopsy of thyroid nodules: comparison of three sets of guidelines. AJR Am J Roentgenol. 2010;194:31-37. 